\title{
The potential of the HAWC Observatory to observe violations of Lorentz Invariance
}

\author{
Lukas Nellen $^{* a}$ for the HAWC collaboration ${ }^{b}$ \\ ${ }^{a}$ I. de Ciencias Nucleares, UNAM, México \\ ${ }^{b}$ For a complete author list, see http://www.hawc-observatory.org/collaboration/icrc2015.php. \\ E-mail: lukas@nucleares.unam.mx
}

\begin{abstract}
The framework of relativistic quantum-field theories requires Lorentz Invariance. Many theories of quantum gravity, on the other hand, include violations of Lorentz Invariance at small scales and high energies. This generates a lot of interest in establishing limits on such effects, and, if possible, observing them directly. Gamma-ray observatories provide a tool to probe parts of the parameter space of models of Lorentz Invariance Violation that is not accessible in terrestrial laboratories and man-made accelerators. Transients, especially gamma-ray bursts, are a particularly promising class of events to search for such phenomena. By combining cosmological distances with high energy emission and short duration, emitting photons up to $30 \mathrm{GeV}$ in less than a second, one can measure the energy dependence of the speed of photons to one part in $10^{16}$. We will discuss the potential of HAWC to detect effects of the violation of Lorentz Invariance and place its sensitivity in the context of existing limits.
\end{abstract}

The 34th International Cosmic Ray Conference,

30 July- 6 August, 2015

The Hague, The Netherlands

${ }^{*}$ Speaker. 


\section{Introduction}

The HAWC Observatory [1,2], located at an elevation of $4100 \mathrm{~m}$ on the volcano Sierra Negra in Mexico, is expected to be capable of placing limits on certain classes of models for quantum gravity that predict the violation of Lorentz Invariance.

Relativistic quantum field theory (RQFT) [3], like many modern theories, requires Lorentz Invariance for consistency. The framework of perturbative RQFT is the basis for the successful Standard Model of particle physics. On the other hand, it has not been possible so far to develop a consistent theory that unifies gravity with RQFTs [4]. Nevertheless, models of Quantum Gravity (QG) have been developed, many of which predict the violation of Lorentz Invariance either at very high energies or very short distances [5]. In many cases, this can result in observable deviations from standard theories [6].

It is typically expected for QG to manifest itself fully at the Planck Scale, set by the Planck Mass $m_{\mathrm{pl}}=\sqrt{\hbar c / G} \approx 10^{19} \mathrm{GeV}$. This makes it also a natural scale at which one expects Lorentz Invariance to be broken. The Planck Scale is not accessible directly in the laboratory or in astrophysical objects in the contemporary universe. It is, however, reasonable to expect that small, residual effects of QG could lead to an observable Lorentz Invariance Violation (LIV) in astroparticle physics, in particular in observations of high energy gamma rays. One of the familiar equations that can get modified is the dispersion relation for photons, resulting in an energy dependent speed for the propagation of photons, instead of a constant speed of light in the vacuum $c$. The leading term in the modified dispersion relation is [7]:

$$
E^{2} \simeq p^{2} c^{2}\left(1+\xi_{n}\left(\frac{p c}{E_{\mathrm{QG}}}\right)^{n}\right)
$$

with $E_{\mathrm{QG}}$ being the energy scale for QG effects, normally the Planck scale, and $\xi_{n}$ a dimensionless expansion coefficient. Depending on details of the model, the leading term is linear $(n=1)$ or quadratic $(n=2)$. Higher order leading terms are generally not considered. When quoting limits, it is common to establish them for a combined scale variable $E_{\mathrm{QG}}^{(n)} \equiv E_{\mathrm{QG}} \xi_{n}^{-1 / n}$. The speed of propagation of photons derived from eq. (1.1) is

$$
v=\frac{\mathrm{d} E}{\mathrm{~d} p} \approx c\left(1+\frac{n+1}{2}\left(\frac{p c}{E_{\mathrm{QG}}^{(n)}}\right)^{n}\right) .
$$

The speed of photons is no longer constant, which means that photons emitted simultaneously will arrive at the observer spread over a time $\Delta t$, which depends on the spread of energy of the photons produced and the distance to the source. For nearby sources, for example pulsars in our galaxy, the curvature of the universe can be neglected when calculating the photon travel time and one obtains for the time spread

$$
\Delta t=\frac{(n+1) d}{2 c} \frac{\Delta E^{n}}{\left(E_{\mathrm{QG}}^{(n)}\right)^{n}} \approx \frac{(n+1) d}{2 c} \frac{E_{\mathrm{max}}^{n}}{\left(E_{\mathrm{QG}}^{(n)}\right)^{n}}
$$

for the distance to source $d$ and $\Delta E^{n}=E_{\max }^{n}-E_{\min }^{n}$. In most cases, the energy range considered spreads several orders of magnitude and one can approximate $\Delta E^{n} \approx E_{\max }^{n}$. 
For photons traveling cosmological distances, for example those originating from Active Galactic Nuclei (AGN) or Gamma Ray Bursts (GRB), one has to take into account the redshift of the source and the non-trivial metric of the universe. The resulting expression for the time spread is [8]

$$
\Delta t=\frac{n+1}{2} H_{0}^{-1} \frac{E_{\max }^{n}}{\left(E_{\mathrm{QG}}^{(n)}\right)^{n}} \int_{0}^{z} \frac{\left(1+z^{\prime}\right)^{n}}{h\left(z^{\prime}\right)} \mathrm{d} z^{\prime}
$$

where $h(z)=\sqrt{\Omega_{\Lambda}+\Omega_{m}(1+z)^{3}}$. The cosmological parameters used in this paper are $H_{0}=$ $70 \mathrm{~km} \mathrm{~s}^{-1} \mathrm{Mpc}^{-1}, \Omega_{\Lambda}=0.7$, and $\Omega_{m}=0.3$. Using the most recent results by Planck [9] does not affect the HAWC reference scenarios significantly.

\section{Limits on the Violation of Lorentz Invariance}

Gamma ray observatories have used observations of pulsars, GRBs, and AGNs to set limits on the scale of QG. A compilation of results can be found in [7], with additional results reported in $[11,15]$. The precise estimate of the propagation delay is a non-trivial part of establishing limits on LIV. A simplifying assumption one can make is that of simultaneous emission of all photons. Doing this, one automatically over-estimates the contribution of LIV to the spread of arrival times $\Delta t$, since one neglects the contribution of astrophysical effects in the source. Improvements in modeling the source and more sophisticated analysis techniques can be used to obtain a better estimate of the contribution of LIV and thereby set more stringent limits.

The current best limits on both the linear and quadratic term have been set by Fermi/LAT's observation of GRB090510 [12]. The limits set are $E_{\mathrm{QG}}^{(1)}>9.1 \cdot 10^{19} \mathrm{GeV}$ and $E_{\mathrm{QG}}^{(2)}>1.3 \cdot 10^{11} \mathrm{GeV}$.

The observation of the flare of PKS 2155-304 on MJD 53944 by H.E.S.S provides the best limits on LIV derived from the observation of AGNs [13]. The reported limit on the quadratic term of $E_{\mathrm{QG}}^{(2)}>6.4 \cdot 10^{10} \mathrm{GeV}$ is within a factor of two of the curent best limit set by Fermi/LAT. The joint analysis by H.E.S.S. and Fermi/LAT of the flaring of PG 1553+113 in April 2012 [14] shows the potential of combining results from multiple observatories, even though the limits are slightly worse than those H.E.S.S obtained from PKS 2155-304.

The best limits derived from pulsars have been set by VERITAS' observation of the pulsation of the Crab [16]. The limits reported are $E_{\mathrm{QG}}^{(1)}>1.7 \cdot 10^{17} \mathrm{GeV}[10]$ and $E_{\mathrm{QG}}^{(2)}>7 \cdot 10^{9} \mathrm{GeV}$ [11].

\section{Potential of HAWC for setting limits on LIV}

We use the sources studied by other observatories in the analyses quoted in section 2 to construct reference scenarios for the HAWC observatory and establish its potential to set limits on LIV.

The scenario for setting limits on LIV from GRBs is motivated by the properties of the short burst GRB090510 [17] and of the long burst GRB130427A [15]. Our reference scenario is a short burst with $\Delta t=1 \mathrm{sec}$ at a redshift of $z=1$, with a maximum observed photon energy of $E_{\max }=100 \mathrm{GeV}$, close to that of GRB130427A [15]. Such a burst is detectable by HAWC if it occurs in its field of view [18]. The time spread and redshift assumed in this scenario are clearly compatible with observed GRBs used to set limits on $\operatorname{LIV}[15,17,19]$. In this scenario, it is 


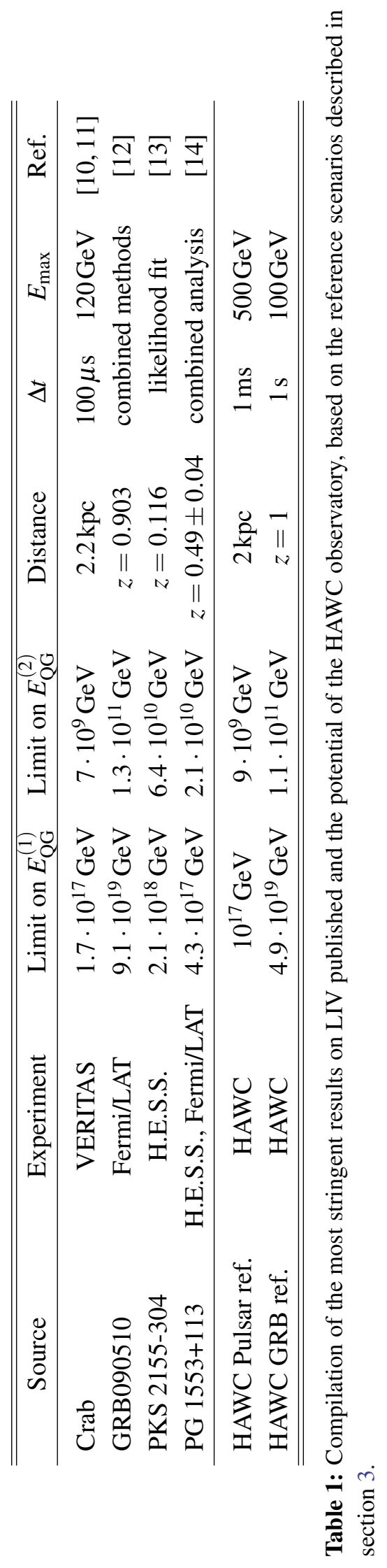


possible for HAWC to set a limit of $4.9 \cdot 10^{19} \mathrm{GeV}$ for the linear term $E_{\mathrm{QG}}^{(1)}$ and $1.1 \cdot 10^{11} \mathrm{GeV}$ for $E_{\mathrm{QG}}^{(2)}$. Comparing these numbers with the limits reported in section 2 shows that HAWC can set competitive limits even with very basic analysis techniques. Improvements to modeling and analysis are expected to allow HAWC to set more stringent limits.

Our reference scenario for pulsars is motivated by the VERITAS observation of the Crab pulsar at energies up to $120 \mathrm{GeV}$ [16] and the limits on LIV derived from that observation [10,11]. We assume a time spread $\Delta t=1 \mathrm{~ms}$, a factor of 10 larger than used in the VERITAS limit. This arbitrary degradation of the resolved time spread was chosen since the potential of HAWC to observe pulsations, and therefore details of the light curve, of the Crab Nebula has not yet been demonstrated. The degenerated time resolution gets compensated by assuming an increased maximum energy of $E_{\max }=500 \mathrm{GeV}$ for a pulsar at a distance of $2 \mathrm{kpc}$. Assuming the observation of such a pulsar, HAWC could establish limits for the linear term $E_{\mathrm{QG}}^{(1)}$ up to $10^{17} \mathrm{GeV}$ and for the quadratic term $E_{\mathrm{QG}}^{(2)}$ of up to $9 \cdot 10^{9} \mathrm{GeV}$. The assumption of an increase in the observed $E_{\max }$ allows for an improved limit on the quadratic term, despite the worsened limit on the time difference. One has to assume that such a source, if it exists, will also be observed by Imaging Air Cherenkov Telescopes (IACTs). It will remain to be seen if the HAWC's ability to monitor a pulsar continuously will be able to compensate for increased detail of observations by IACTs.

It is unlikely that HAWC will be able to improve on limits derived from the observation of flaring AGNs by IACTs. The lower statistics of HAWC results in less detail in light curves, which in turn results in a less stringent estimate of $\Delta t$.

Of the sources considered here, one-shot transients like GRBs provide the most favourable objects for setting stringent limits on LIV. HAWC can reach higher in energy than satellites like Fermi and has a larger probability to see a GRB in its field of view than an IACT. Combining observations by HAWC with satellite based observations has the potential to provide even better limits on LIV. Pulsars have the advantage that they are long-lived and therefore reliably detectable, even though HAWC's ability to observe pulsation still has to be established. The large field of view and high duty cycle of HAWC results in a large accumulated time on each source, which can make up for the detail in the high statistics but time-limited observations by IACTs.

\section{Conclusions}

We developed scenarios establishing the potential of the HAWC Observatory to set limits on LIV in models with modified speed of propagation of photons. Using observations of GRBs, HAWC has the potential to set competitive limits. So far, we consider only very simple scenarios and analysis techniques. We expect to improve the potential of HAWC in this area in the future.

\section{Acknowledgments}

We acknowledge the support from: the US National Science Foundation (NSF); the US Department of Energy Office of High-Energy Physics; the Laboratory Directed Research and Development (LDRD) program of Los Alamos National Laboratory; Consejo Nacional de Ciencia y Tecnología (CONACyT), Mexico (grants 260378, 55155, 105666, 122331, 132197, 167281, 167733); Red de Física de Altas Energías, Mexico; DGAPA-UNAM (grants IG100414-3, IN108713, IN121309, IN115409, IN111315); VIEP-BUAP (grant 161-EXC-2011); the University of Wisconsin Alumni 
Research Foundation; the Institute of Geophysics, Planetary Physics, and Signatures at Los Alamos National Laboratory; the Luc Binette Foundation UNAM Postdoctoral Fellowship program.

\section{References}

[1] HAWC Collaboration, J. Pretz, Highlights from the High Altitude Water Cherenkov Observatory, in Proc. 34th ICRC, (The Hague, The Netherlands), August, 2015.

[2] http: /www.hawc-observatory . org.

[3] M. E. Peskin and D. V. Schroeder, An Introduction To Quantum Field Theory. Westview Press, 1995.

[4] R. Woodard, How Far Are We from the Quantum Theory of Gravity?, Rept.Prog.Phys. 72 (2009) 126002, [arXiv:0907.4238].

[5] L. Smolin, How far are we from the quantum theory of gravity?, hep-th/ 0303185.

[6] D. Mattingly, Modern tests of Lorentz invariance, Living Rev.Rel. 8 (2005) 5, [gr-qc/ 0502097 ].

[7] J. Bolmont and A. Jacholkowska, Lorentz Symmetry Breaking studies with photons from Astrophysical Observations, Adv.Space Res. 47 (2011) 380-391, [arXiv:1 007.4954 ].

[8] J. Bolmont, J.-L. Atteia, A. Jacholkowska, F. Piron, and G. Pizzichini, Study of time lags in HETE-2 Gamma-Ray Bursts with redshift: search for astrophysical effects and Quantum Gravity signature, Astrophys.J. 676 (2008) 532-544, [astro-ph/ 060372 5].

[9] Planck Collaboration, P. Ade et al., Planck 2015 results. XIII. Cosmological parameters, arXiv: 1502.0158.

[10] VERITAS Collaboration, B. Zitzer, Lorentz Invariance Violation Limits from the Crab Pulsar using VERITAS, arXiv:1307.8382.

[11] A. N. Otte, Prospects of performing Lorentz invariance tests with VHE emission from pulsars, arXiv:1208.2033.

[12] V. Vasileiou, A. Jacholkowska, F. Piron, J. Bolmont, C. Couturier, et al., Constraints on Lorentz Invariance Violation from Fermi-Large Area Telescope Observations of Gamma-Ray Bursts, Phys.Rev. D87 (2013), no. 12 122001, [arXiv: 1305. 3463].

[13] HESS Collaboration, A. Abramowski et al., Search for Lorentz Invariance breaking with a likelihood fit of the PKS 2155-304 Flare Data Taken on MJD 53944, Astropart.Phys. 34 (2011) 738-747, [arXiv:1101.3650].

[14] Fermi, HESS Collaboration, D. Sanchez, F. Brun, C. Couturier, A. Jacholkowska, and J.-P. Lenain, Probe of Lorentz Invariance Violation effects and determination of the distance of PG 1553+113, arXiv: 1502.0292.

[15] G. Amelino-Camelia, F. Fiore, D. Guetta, and S. Puccetti, Quantum-spacetime scenarios and soft spectral lags of the remarkable GRB130427A, Adv.High Energy Phys. 2014 (2014) 597384, [arXiv:1305.2626].

[16] VERITAS Collaboration, E. Aliu et al., Detection of Pulsed Gamma Rays Above 100 GeV from the Crab Pulsar, Science 334 (2011) 69-72, [arXiv:1108.3797].

[17] Fermi-LAT, Fermi GBM Collaboration, A. A. Abdo, M. Ackermann, M. Ajello, K. Asano, W. B. Atwood, M. Axelsson, L. Baldini, J. Ballet, G. Barbiellini, M. G. Baring, and et al., A limit on the variation of the speed of light arising from quantum gravity effects, Nature 462 (Nov., 2009) 331-334, [arXiv:0908.1832]. 
[18] HAWC Collaboration, A. Abeysekara et al., On the sensitivity of the HAWC observatory to gamma-ray bursts, Astropart.Phys. 35 (2012) 641-650, [arXiv:1108.6034].

[19] Fermi-LAT, Fermi GBM Collaboration, A. A. Abdo et al., Fermi Observations of High-Energy Gamma-Ray Emission from GRB 080916C, Science 323 (2009) 1688-1693. 